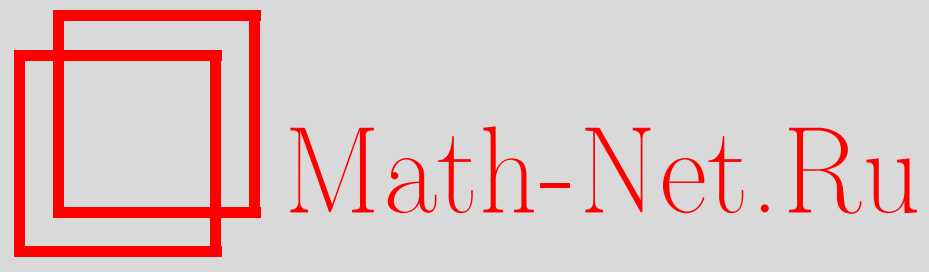

А. Г. Эршлер, Обобщенные броуновские петли, УМН, 2007, том 62, выпуск 3, 221-222

DOI: https://doi.org/10.4213/rm7042

Использование Общероссийского математического портала Math-Net.Ru подразумевает, что вы прочитали и согласны с пользовательским соглашением http: //www . mathnet.ru/rus/agreement

Параметры загрузки:

IP : 44.207 .124 .84

26 апреля 2023 г., $11: 32: 33$

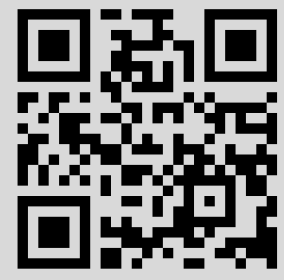




\section{Обобщенные броуновские петли}

\section{А. Г. Эршлер}

Мы определяем и изучаем случайные вложения конечных нормализованных графов в графы и группы. Рассмотрим связный конечный граф Г с выделенной точкой $O$ и граф $G$ с выделенной точкой $e$. В этой заметке мы рассматриваем случай, когда $G$ является графом Кэли бесконечной конечно порожденной группы.

Пусть $O=X_{1}, X_{2}, \ldots, X_{k}$ - это вершины графа Г. Для любого $n$ мы рассматриваем граф $\Gamma^{(n)}$, который получается из $Г$ умножением длины каждого ребра на $n$ и добавлением $n-1$ дополнительных вершин на каждое ребро таким образом, что длина каждого ребра в $\Gamma^{(n)}$ снова становится равной единице. Вершины $\Gamma^{(n)}$, соответствующие $X_{1}, \ldots, X_{k}$, мы называем главными вершинами и обозначаем их $O^{(n)}=X_{1}^{(n)}$, $X_{2}^{(n)}, \ldots, X_{k}^{(n)}$.

Случайные вложения $\Gamma^{(n)}$ в $G$. Пусть $\rho: \Gamma^{(n)} \rightarrow G$ - это сохраняющее ребра вложение такое, что $\rho\left(O^{(n)}\right)=e$. Мы рассматриваем равномерное вероятностное распределение на таких вложениях и изучаем предельное распределение расстояний между образами главных вершин $\rho\left(X_{1}^{(n)}\right), \rho\left(X_{2}^{(n)}\right), \ldots, \rho\left(X_{k}^{(n)}\right) \subset G^{k}$. В дальнейшем мы предполагаем, что $G$ имеет петлю в вершине $e$. Можно не делать это предположение и рассматривать только четные $n$.

Заметим, что для регулярного графа $G$ вероятность $\mathrm{P}\left[\phi\left(X_{i}^{(n)}\right)=g_{i}\right.$ для всех $i$ : $1 \leqslant i \leqslant k]$ пропорциональна произведению $\prod p_{n}\left(g_{i}, g_{j}\right)$ по всем $i, j$ таким, что $X_{i}, X_{j}$ соединены ребром в $Г$. Здесь $g_{1}=e, g_{2}, \ldots, g_{k}$ - это вершины в $G$ и $p_{n}(g, h)$ - вероятность перехода из $g$ в $h$ за $n$ шагов простого случайного блуждания на $G$.

Средний диаметр образа главных вершин мы обозначаем $\operatorname{diam}_{G, e, O}(\Gamma, n)$. Нетрудно увидеть, что для графа Кэли конечно порожденной группы $G$ распределение расстояний между образами главных вершин не зависит от выбора $O$ в $\Gamma$ и $e \in G$. Поэтому в этой ситуации далее мы будем опускать в обозначениях индексы $O$ и $e$.

Сохраняющие ребра отображения между графами называются также гомоморфизмами графов. Случайные гомоморфизмы ненормализованных конечных графов в $\mathbb{Z}$ изучались в [1]-[3]. В этих статьях случайные вложения графа $Г$ называются Г-индексированными случайными блужданиями. Заметим, что в случае рассматриваемых нами случайных вложений нормализованных графов аналогия со случайными блужданиями еще сильнее.

Пусть $G$ - регулярный граф конечной валентности. Если $\Gamma=\Gamma_{r w}-$ это граф, имеющий две вершины $X_{1}$ и $X_{2}$ и одно ребро между ними, то распределение $\rho\left(X_{2}^{(n)}\right)$ это распределение $n$-го шага простого случайного блуждания по $G$, начинающегося в $e$. Если $\Gamma=\Gamma_{B b}-$ это граф, имеющий две вершины $X_{1}, X_{2}$ и два ребра между ними, тогда $\rho\left(X_{2}^{(n)}\right)$ распределена как средняя точка случайной петли в $G$ (с начальной и конечной точкой в $e$ ). Случайные петли в группах изучались в [4]-[8]. Можно показать, что для нильпотентных (в частности, для абелевых) групп асимптотическое поведение вложений конечных графов сильно напоминает два вышеупомянутых случая: случайных блужданий и случайных петель. Из следующих теорем видно, что в общем случае ситуация может быть существенно иной.

Рассмотрим конечный граф Г, имеющий по крайней мере две вершины. Напомним, что минимальный разрез mincut $_{\Gamma}$ графа $Г$ - это минимальное число ребер, после удаления которых $\Gamma$ перестает быть связным. Для пары различных вершин $x$ и $y$ в графе Г минимальный разрез $\operatorname{mincut}_{\Gamma}(x, y)$ между ними - это минимальное число ребер, после удаления которых $x$ и $y$ оказываются в разных компонентах связности. Ребро связного графа называется разделяющим, если после его удаления граф теряет связность. 
Теорема 1. Рассмотрим свободную группу $G=F_{m}$ с $m \geqslant 2$ образующими, $S_{m}-$ множество образующих, свободно порождающих $F_{m}, u S=S_{m} \cup\{e\}$. Пусть Г это конечный связный граф, $x$ и $y$-две различные вершины графа Г.

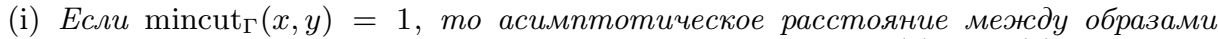
главных вершин, соответствующих х и $y$, линейно: $\mathrm{E}\left[d_{G}\left(\phi\left(X^{(n)}\right), \phi\left(Y^{(n)}\right)\right)\right] \sim n$.

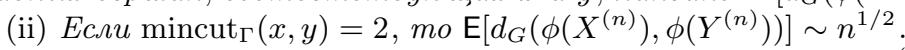

(iii) В остальных случалх (если $\left.\operatorname{mincut}_{\Gamma}(x, y) \geqslant 3\right) \mathrm{E}\left[d_{G}\left(\phi\left(X^{(n)}\right), \phi\left(Y^{(n)}\right)\right)\right] \sim$ const.

Наиболее простой частный случай второго пункта теоремы - случайные петли в свободной группе. Этот случай был известен ранее (см. [7], [4]).

Напомним, что сплетение групп $A$ и $B$ (обозначаемое $A$ 乙 $B$ ) - это полупрямое произведение $A$ и $\sum_{A} B$ для действия сдвигами $A$ на $\sum_{A} B$ : для $a \in A$ и отображения $f: A \rightarrow B$ такого, что $f \in \sum_{A} B$, выполнено равенство $f^{a}(x)=f\left(a^{-1} x\right), x \in A$. Сплетение конечно порожденных групп тоже конечно порождено. В следующей теореме мы описываем асимптотический диаметр конечных графов для сплетения $\mathbb{Z} \mathrm{c}$ конечной группой (для любой системы образующих в таком сплетении).

Теорема 2. Пусть $G=\mathbb{Z} \imath A, A$ - конечная группа, содержащая по крайней мере два элемента, а $S$ - конечнал симметричная система образующих группы $G$, содержащая нейтральный элемент.

Рассмотрим конечный связный граф Г, имеющий по крайней мере две вершины.

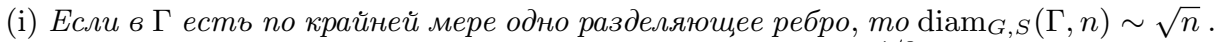

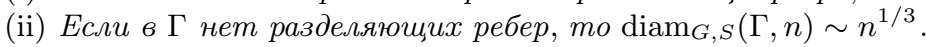

Опять же, частный случай второй части теоремы 2 - это случайные петли в рассматриваемом сплетении. Это первый известный пример случайных петель на группе, размер которых не асимптотически эквивалентен $n^{1 / 2}$ (см. [5]).

Для изучения асимптотик, связанных со случайными вложениями нормированных графов в граф $G$, полезно знать достаточно точные оценки на переходные вероятности для случайного блуждания на $G$. В доказательстве теоремы 1 мы, в частности, пользуемся оценками Лэли для переходных вероятностей на свободных группах [9]. При доказательстве теоремы 2 мы используем следующую лемму.

Лемма. Рассмотрим $G, A$ u $S$ такие, как в теореме 2. Существуют $C, D>0$ такие, что для любого натурального $n$ и для любого $g \in G$ вида $g=(0, f)$, имеюощего длину менъше $C n^{1 / 3}$, выполняется следующее неравенство для переходных вероятностей простого случайного блуждания $(G, S): p_{n}(e, g) \geqslant D p_{n}(e, e)$.

Для определенного выбора $S$ утверждение леммы (без предположения $g=(e, f)$ ) было доказано ранее Д. Ревелем [10].

\section{Список литературы}

[1] I. Benjamini, O. Höggström, E. Mossel, J. Combin. Theory Ser. B, 78:1 (2000), 86-114. [2] I. Benjamini, G. Schechtman, Random Structures Algorithms, 17:1 (2000), 20-25. [3] J. Kahn, Israel J. Math., 124 (2001), 189-201. [4] P. Bougerol, T. Jeulin, Probab. Theory Related Fields, 115:1 (1999), 95-120. [5] A. Erschler, Probab. Theory Related Fields, 136:4 (2006), 560-586. [6] А. В. Лётчиков, УМH, 51:1 (1996), 51-100. [7] S. K. Nechaev, A. Yu. Grosberg, A. M. Vershik, J. Phys. A, 29:10 (1996), 2411-2433. [8] S. K. Nechaev, Ya. G. Sinai, Bol. Soc. Brasil. Mat. (N.S.), 21:2 (1991), 121-132. [9] S. P. Lalley, J. Theoret. Probab., 4:4 (1991), 701-723. [10] D. Revelle, Electron. Comm. Probab., 8 (2003), 142-154.

А. Г. Эршлер (А. G. Erschler)

Université Paris XI (Orsay);

Санкт-Петербургское отделение

Математического института им. В. А. Стеклова РАН

E-mail: Anna.Erschler@math.u-psud.fr
Представлено В. М. Бухштабером Принято редколлегией 30.03.2007 